\title{
Adjustment of Single and Double Braced Quadrilateral using Least Square Condition Equation
}

\author{
R. Ehigiator-Irughe and B. M. Mohammad Muhajir
}

\section{ABSTRACT}

Quadrilateral is a single or double triangulation system consisting of figures with four corners stations. It consists of two known stations and a base line. Other corners of the triangles are measured using precise instrument. The systems is treated as the strongest with the best arranged triangular structure which provides adequate means of determining the lengths of other sides of the triangle whose length, bearings and positions are required. In this four sided polygon with four (4) points were established. The coordinate of two points ( $A$ and $B$ ) are known while the coordinates of points $(C$ and $D$ ) are required. The purpose of the exercise was to use least square condition equation to determine and adjust coordinates of the two unknown points using the Angular measurements of quadrilateral. Two separate measurements were taken (observation 1 and observation 2) forming a network of a single and double braced quadrilateral respectively. After which, the data obtained were reduced and then adjusted using least square condition equation.

Keywords: Quadrilateral, Condition Equation, Observation, normal equation, Lagrange.

\section{INTRODUCTION}

Horizontal control has been performed using our classical Geomatics Engineering approaches. Some of the approaches include but not limited to; Traversing, Trilateration, Triangulation and recently Global navigation Satellite systems (GNSS). One of the methods of triangulation is the subdivision of complex figure into smaller triangles as either single or double quadrilateral. The purpose is to determine the coordinates of unknown points using least square method.

Geomatics measurements are usually inseparable with errors during field observations and these errors are due to many factors, which include but not limited to human, natural and equipment used etc. The errors cannot be left in the final data without finding a way to eliminate them; this gave reason for the use of mathematical adjustment procedure [1]. In the first half of the 19th century the Least Squares (LS) adjustment technique was developed [2]. LS are the conventional technique for adjusting Geomatics measurements till date. The LS technique minimizes the sum of the squares of differences between the observation and estimate [3]. Apart from LS other methods of adjusting Geomatics methods have been developed, such as Kalman Filter (KF) [4], Least Squares Collocation (LSC) [5] and Total Least Squares (TLS) [6] - [9]. This work will expound in its simplest form fundamental methods of LS adjustment equation as it applies to single and double Quadrilateral Triangular figure using condition equation method.

\section{Conditional Least SQuares AdJustment}

The general form of the Conditional mathematical model given by:

$$
B_{r, n} v_{n, 1}+\Delta_{r, 1}=0
$$

where $\mathrm{B}$ is the deigned matrix,

$\mathrm{V}$ is the vector,

Dimension $r$ is the redundancy,

dimension $\mathrm{n}$ is the number of given observations

$\Delta$ is the vector of misclosure.

In equation (1) $r$ (degrees of freedom $)=n-n o$.

Two basic properties must be satisfied for the conditional model:

1) Number of equations $=$ Number of degrees of freedoms. This means that each redundant observation provides one independent condition equation.

2) The equations describe the functional relationship among the observations only. This obviously indicates that the unknown parameters $\mathrm{x}$ will not be among the direct output of the conditional adjustment. Thus the adjusted parameters

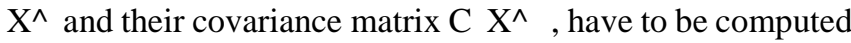
after the adjustment using the direct model $\left(\mathrm{X}^{\wedge}=\mathrm{f}\left({ }^{\wedge} \mathrm{I}\right)\right)$ and the law of propagation of variances (this is a disadvantage when comparing the conditional and the parametric adjustment). 
A. Adjustment of a single braced quadrilateral using least square condition equation

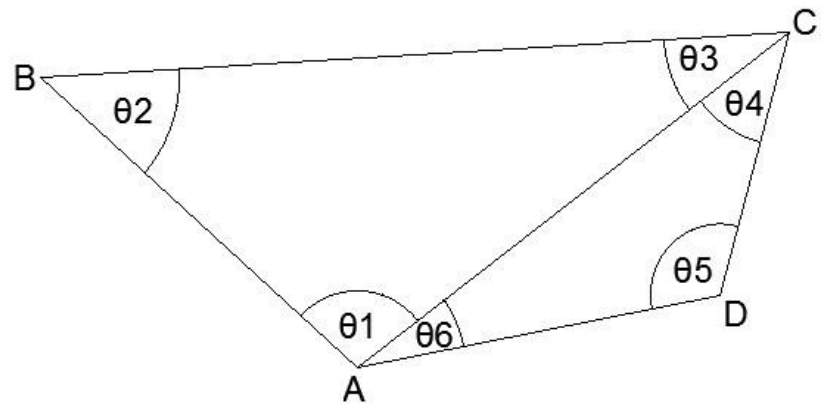

Fig. 1: Single Braced Quadrilateral.

The field measurements for a single braced quadrilateral $\mathrm{ABCD}$ are given below. Using this data, we will compute the coordinates of station $\mathrm{C}$ and $\mathrm{D}$.

TABLE 1: FIELD OBSERVATION DATA

\begin{tabular}{|c|c|c|c|c|c|c|}
\hline $\begin{array}{l}\text { ANGLE } \\
\qquad(\theta)\end{array}$ & $\begin{array}{c}\text { STN } \\
\text { FROM }\end{array}$ & FACE & Degree & mins & $\mathrm{sec}$ & $\begin{array}{c}\text { STN } \\
\text { TO } \\
\end{array}$ \\
\hline \multirow{4}{*}{1} & \multirow{4}{*}{ A } & $\mathrm{L}$ & 00 & 00 & 00 & $\mathrm{D}$ \\
\hline & & $\mathrm{L}$ & 19 & 11 & 23 & $\mathrm{C}$ \\
\hline & & $\mathrm{R}$ & 199 & 11 & 24 & $\mathrm{C}$ \\
\hline & & $\mathrm{R}$ & 180 & 00 & 02 & $\mathrm{D}$ \\
\hline \multirow{4}{*}{6} & \multirow{4}{*}{ A } & $\mathrm{L}$ & 0 & 00 & 00 & $\mathrm{C}$ \\
\hline & & $\mathrm{L}$ & 29 & 58 & 13 & B \\
\hline & & $\mathrm{R}$ & 209 & 58 & 12 & B \\
\hline & & $\mathrm{R}$ & 179 & 59 & 58 & $\mathrm{C}$ \\
\hline \multirow{4}{*}{2} & \multirow{4}{*}{ B } & $\mathrm{L}$ & 0 & 00 & 00 & $\mathrm{D}$ \\
\hline & & $\mathrm{L}$ & 117 & 37 & 01 & A \\
\hline & & $\mathrm{R}$ & 297 & 37 & 03 & A \\
\hline & & $\mathrm{R}$ & 180 & 00 & 01 & $\mathrm{D}$ \\
\hline \multirow{4}{*}{3} & \multirow{4}{*}{ B } & $\mathrm{L}$ & 0 & 00 & 00 & $\mathrm{D}$ \\
\hline & & $\mathrm{L}$ & 43 & 11 & 22 & $\mathrm{C}$ \\
\hline & & $\mathrm{R}$ & 223 & 11 & 29 & $\mathrm{C}$ \\
\hline & & $\mathrm{R}$ & 179 & 59 & 59 & $\mathrm{D}$ \\
\hline \multirow{4}{*}{4} & \multirow{4}{*}{$\mathrm{C}$} & $\mathrm{L}$ & 0 & 00 & 00 & A \\
\hline & & $\mathrm{L}$ & 48 & 21 & 31 & $\mathrm{~B}$ \\
\hline & & $\mathrm{R}$ & 228 & 21 & 33 & $\mathrm{~B}$ \\
\hline & & $\mathrm{R}$ & 180 & 00 & 03 & A \\
\hline \multirow{4}{*}{5} & \multirow{4}{*}{$\mathrm{C}$} & $\mathrm{L}$ & 0 & 00 & 00 & A \\
\hline & & $\mathrm{L}$ & 101 & 40 & 08 & $\mathrm{D}$ \\
\hline & & $\mathrm{R}$ & 281 & 40 & 07 & $\mathrm{D}$ \\
\hline & & $\mathrm{R}$ & 180 & 00 & 01 & A \\
\hline
\end{tabular}

TABLE 3: OBSERVED ANGLES AND STANDARD ERRORS

\begin{tabular}{|c|c|c|c|c|c|}
\hline $\begin{array}{c}\text { ANGLE } \\
(\theta)\end{array}$ & $\begin{array}{c}\text { OBS. VALUE } \\
(\theta)\end{array}$ & $\begin{array}{c}\text { STD } \\
\text { ERROR } \\
\left(\sigma^{2}\right)\end{array}$ & Station & Eastings & Northings \\
\cline { 1 - 4 } 1 & $1^{\circ} 11^{\prime} 22.5^{\prime \prime}$ & 0.7 & A & 507327.641 & 91756.439 \\
\hline 2 & $117^{\circ} 37^{\prime} 1.5^{\prime \prime}$ & 0.7 & B & 507450.410 & 92079.322 \\
\cline { 1 - 3 } 3 & $43^{\circ} 11^{\prime} 26.0^{\prime \prime}$ & 5.7 & & & \\
\cline { 1 - 3 } 4 & $48^{\circ} 21^{\prime} 30.5^{\prime \prime}$ & 0.7 & & & \\
\cline { 1 - 3 } 5 & $101^{\circ} 40^{\prime} 7.0^{\prime \prime}$ & 1.4 & & & \\
\cline { 1 - 3 } 6 & $29^{\circ} 58^{\prime} 13.5^{\prime \prime}$ & 0.7 & & & \\
\hline
\end{tabular}

The number of total observations $(n)=6$ Angles.

No of sides $(\mathrm{S})=4$.

The number of necessary observations $\left(\mathrm{n}_{0}\right)=2$ (No of new points $)=2(S-2)=4$ Hence, the number of conditions $(r)=n$ $-\mathrm{n}_{0}=2$.

\section{Condition equations}

There are two condition equation:

$$
\begin{gathered}
\mathrm{V} 1+\mathrm{V} 2+\mathrm{V} 3=180^{\circ}-(\theta 1+\theta 2+\theta 3)=180^{\circ}-179^{\circ} 59^{\prime} 50.0^{\prime \prime}=\Delta 1 \\
\Delta 1=10.0^{\prime \prime} \\
\mathrm{V} 4+\mathrm{V} 5+\mathrm{V} 6=180^{\circ}-(\theta 4+\theta 5+\theta 6)=180^{\circ}-179^{\circ} 59^{\prime} 51.0^{\prime \prime}=\Delta 2, \\
\Delta 2=9.0^{\prime \prime}
\end{gathered}
$$

The design matrix is given as:

$$
B=\left[\begin{array}{llllll}
1 & 1 & 1 & 0 & 0 & 0 \\
0 & 0 & 0 & 1 & 1 & 1
\end{array}\right]
$$

The vector is given as:

$$
v=\left[\begin{array}{l}
v 1 \\
v 2 \\
v 3 \\
v 4 \\
v 5 \\
v 6
\end{array}\right]
$$

The general for is given as:

$$
[B][V]=[\Delta],
$$

we have from equation (1)

$$
\left[\begin{array}{llllll}
1 & 1 & 1 & 0 & 0 & 0 \\
0 & 0 & 0 & 1 & 1 & 1
\end{array}\right]\left[\begin{array}{l}
v 1 \\
v 2 \\
v 3 \\
v 4 \\
v 5 \\
v 6
\end{array}\right]=\left[\begin{array}{c}
\Delta 1 \\
\Delta 2
\end{array}\right]
$$

and the weight matrix is:

$$
\begin{aligned}
& W=\operatorname{diag}\left[1 / \sigma_{11}^{2}, 1 / \sigma_{22}^{2}, 1 / \sigma_{33}^{2}, 1 / \sigma_{44}^{2}, 1 / \sigma_{55}^{2}, 1 / \sigma_{66}^{2}\right] \\
& W^{-1}=\left[\sigma_{11}^{2}{ }^{\prime} \sigma_{22}^{2}{ }^{\prime} \sigma_{33}^{2}{ }^{\prime} \sigma_{44}^{2}{ }^{\prime} \sigma_{55}^{2}{ }^{\prime} \sigma_{66}^{2}{ }^{\prime}\right] \\
& W^{-1}=\left[\begin{array}{cccccc}
1.43 & 0 & 0 & 0 & 0 & 0 \\
0 & 1.43 & 0 & 0 & 0 & 0 \\
0 & 0 & 0.18 & 0 & 0 & 0 \\
0 & 0 & 0 & 1.43 & 0 & 0 \\
0 & 0 & 0 & 0 & 0.71 & 0 \\
0 & 0 & 0 & 0 & 0 & 1.43
\end{array}\right]
\end{aligned}
$$


TABLE 2: ANGULAR REDUCTION AND DETERMINATION OF STANDARD ERROR $(\sigma)$

\begin{tabular}{|c|c|c|c|c|c|c|c|}
\hline \multirow{2}{*}{$\begin{array}{l}\text { ANGLE } \\
\qquad(\theta)\end{array}$} & \multirow[b]{2}{*}{$\mathrm{L}$} & \multirow[b]{2}{*}{$\mathrm{R}$} & \multirow[b]{2}{*}{ Mean $\left(\theta_{m}\right)$} & \multicolumn{3}{|c|}{$\theta_{m}-\theta_{i}$} & \multirow{2}{*}{$\begin{array}{c}\text { Std. error } \\
\qquad\left(\sigma^{2}\right)\end{array}$} \\
\hline & & & & $\mathrm{V}=\theta_{m}-\theta_{l}$ & $\mathrm{~V}=\theta_{m}-\theta_{r}$ & $\left(\frac{\theta_{m}-\theta_{r}}{\theta_{m}-\theta_{l}}\right) / 2$ & \\
\hline 1 & $19^{\circ} 11^{\prime} 23^{\prime \prime}$ & $19^{\circ} 11^{\prime} 22^{\prime \prime}$ & $19^{\circ} 11^{\prime} 22.5^{\prime \prime}$ & $0.50 "$ & $0.50 "$ & $0.5^{\prime \prime}$ & 0.7 \\
\hline 6 & $29^{\circ} 58^{\prime} 13^{\prime \prime}$ & $29^{\circ} 58^{\prime} 14^{\prime \prime}$ & $29^{\circ} 58^{\prime} 13.5^{\prime \prime}$ & $0.50 "$ & $0.50^{\prime \prime}$ & $0.5^{\prime \prime}$ & 0.7 \\
\hline 2 & $117^{\circ} 37^{\prime} 1^{\prime \prime}$ & $117^{\circ} 37^{\prime} 2^{\prime \prime}$ & $117^{\circ} 37^{\prime} 1.5^{\prime \prime}$ & $0.50 "$ & $0.50 "$ & $0.5^{\prime \prime}$ & 0.7 \\
\hline 3 & $43^{\circ} 11^{\prime} 22^{\prime \prime}$ & $43^{\circ} 11^{\prime} 30^{\prime \prime}$ & $43^{\circ} 11^{\prime} 26.0^{\prime \prime}$ & $4.00^{\prime \prime}$ & $4.00^{\prime \prime}$ & $4.00^{\prime \prime}$ & 5.7 \\
\hline 4 & $48^{\circ} 21^{\prime} 31^{\prime \prime}$ & $48^{\circ} 21^{\prime} 30^{\prime \prime}$ & $48^{\circ} 21^{\prime} 30.5^{\prime \prime}$ & $0.50 "$ & $0.50 "$ & $0.5^{\prime \prime}$ & 0.7 \\
\hline 5 & $101^{\circ} 40^{\prime} 8^{\prime \prime}$ & $101^{\circ} 40^{\prime} 6^{\prime \prime}$ & $101^{\circ} 40^{\prime} 7.0^{\prime \prime}$ & $1.00^{\prime \prime}$ & $1.00^{\prime \prime}$ & $1.0 "$ & 1.4 \\
\hline
\end{tabular}

Where $\sigma^{2}=\left[\left(\sqrt{v_{m}^{2}-v_{i}^{2}}\right) / r_{n}-1\right]=\left[\left(\sqrt{\left(\frac{\theta_{m}-\theta_{r}}{\theta_{m}-\theta_{l}}\right) / 2}\right) / r_{n}-1\right], r_{n}=2$

2. The solution to the normal equations is given as:

$$
M=\left[B \times W^{-1} \times B^{T}\right]
$$$$
\begin{gathered}
M=\left[\begin{array}{llllll}
1 & 1 & 1 & 0 & 0 & 0 \\
0 & 0 & 0 & 1 & 1 & 1
\end{array}\right]\left[\begin{array}{cccccc}
1.43 & 0 & 0 & 0 & 0 & 0 \\
0 & 1.43 & 0 & 0 & 0 & 0 \\
0 & 0 & 0.18 & 0 & 0 & 0 \\
0 & 0 & 0 & 1.43 & 0 & 0 \\
0 & 0 & 0 & 0 & 0.71 & 0 \\
0 & 0 & 0 & 0 & 0 & 1.43
\end{array}\right]\left[\begin{array}{ll}
1 & 0 \\
1 & 0 \\
1 & 0 \\
0 & 1 \\
0 & 1 \\
0 & 1
\end{array}\right] \\
M=\left[\begin{array}{ll}
3.04 & 0 \\
0 & 3.57
\end{array}\right]
\end{gathered}
$$

\section{The Lagrange}

The vector of misclosure is given as:

$$
\begin{aligned}
& \Delta=\left[\begin{array}{l}
10 \\
9
\end{array}\right] \\
& K=\left[M^{-1} \times \Delta\right] \\
& K=\left[\begin{array}{lc}
3.04 & 0 \\
0 & 3.57
\end{array}\right]\left[\begin{array}{l}
10 \\
9
\end{array}\right] \\
& K=\left[\begin{array}{l}
3.2895^{\prime \prime} \\
2.5210^{\prime \prime}
\end{array}\right]
\end{aligned}
$$

4. The residuals are given as:

$$
\begin{aligned}
& V=W^{-1} B^{T} K \\
& V=\left[\begin{array}{l}
4.7039^{\prime \prime} \\
4.7039^{\prime \prime} \\
0.5921^{\prime \prime} \\
3.6050^{\prime \prime} \\
1.7899^{\prime \prime} \\
3.6050^{\prime \prime}
\end{array}\right]
\end{aligned}
$$

Variance

$$
\begin{aligned}
\sigma^{2} & =\left[\frac{V^{T} V}{r}\right] \\
\sigma^{2} & =[18.4503]
\end{aligned}
$$

5. The adjusted observations

Finally, adding the residuals to the observations, we obtain the adjusted observations: $\bar{\imath}=\mathrm{L}+\mathrm{V}$

TABLE 4: ADJUSTED OBSERVATIONS

\begin{tabular}{|c|c|c|c|}
\hline $\begin{array}{c}\text { ANGLE } \\
(\theta)\end{array}$ & $\begin{array}{c}\text { OBSERVED } \\
\text { ANGLE }(\theta)\end{array}$ & Residuals (v) & $\begin{array}{c}\text { CORRECTED } \\
\text { ANGLE }(\theta)\end{array}$ \\
\hline 1 & $1^{\circ} 11^{\prime} 22.5^{\prime \prime}$ & $4.7039^{\prime \prime}$ & $1^{\circ} 11^{\prime} 27.20^{\prime \prime}$ \\
\hline 2 & $117^{\circ} 37^{\prime} 1.5^{\prime \prime}$ & $4.7039^{\prime \prime}$ & $117^{\circ} 37^{\prime} 6.20^{\prime \prime}$ \\
\hline 3 & $43^{\circ} 11^{\prime} 26.0^{\prime \prime}$ & $0.5921^{\prime \prime}$ & $43^{\circ} 11^{\prime} 26.59^{\prime \prime}$ \\
\hline 4 & $48^{\circ} 21^{\prime} 30.5^{\prime \prime}$ & $3.6050^{\prime \prime}$ & $48^{\circ} 21^{\prime} 34.10^{\prime \prime}$ \\
\hline 5 & $101^{\circ} 40^{\prime} 7.0^{\prime \prime}$ & $1.7899^{\prime \prime}$ & $101^{\circ} 40^{\prime} 8.8^{\prime \prime}$ \\
\hline 6 & $29^{\circ} 58^{\prime} 13.5^{\prime \prime}$ & $3.6050^{\prime \prime}$ & $29^{\circ} 58^{\prime} 17.11^{\prime \prime}$ \\
\hline$\Sigma$ & $359^{\circ} 59^{\prime} 41.0^{\prime \prime}$ & $19.00^{\prime \prime}$ & $360^{\circ} 0^{\prime} 0.00^{\prime \prime}$ \\
\hline
\end{tabular}

6. Azimuth of lines:

Bearing of line,

$$
\beta=\tan ^{-1}(\mathrm{X} 2-\mathrm{X} 1) /(\mathrm{Y} 2-\mathrm{Y} 1)
$$

1. $\quad \beta(\mathrm{AB})=\tan ^{-1}(507,450.410-507,327.641) /(92,079.322$ $-91,756.439)=20^{\circ} 49^{\prime} 5.58^{\prime \prime}$

$\operatorname{ANGLE}(\theta 2)=117^{\circ} 37^{\prime} 6.2^{\prime \prime}$

2. $\beta(\mathrm{BC})=\beta(\mathrm{BA})-\theta 2=20^{\circ} 49^{\prime} 5.58^{\prime \prime}+180-117^{\circ} 37^{\prime} 6.2^{\prime \prime}=$ $=83^{\circ} 11^{\prime} 59.38^{\prime \prime}$

ANGLE $(\theta 3+\theta 4)=43^{\circ} 11^{\prime} 26.59^{\prime \prime}+48^{\circ} 21^{\prime} 34.10^{\prime \prime}=91^{\circ} 33^{\prime} 0.69^{\prime \prime}$

3. $\beta(\mathrm{CD})=\beta(\mathrm{CB})-(\theta 3+\theta 4)=83^{\circ} 11^{\prime} 59.38^{\prime \prime}+180-91^{\circ} 33^{\prime}$ $7.71^{\prime \prime}=171^{\circ} 38^{\prime} 58.69^{\prime \prime}$

4. $\beta(\mathrm{AC})=\beta(\mathrm{AB})+\theta 1=20^{\circ} 49^{\prime} 5.58^{\prime \prime}+19^{\circ} 11^{\prime} 27.20^{\prime \prime}=$ $=40^{\circ} 00^{\prime} 32.78^{\prime \prime}$

$\operatorname{ANGLE}(\theta 5)=101^{\circ} 40^{\prime} 8.80^{\prime \prime}$.

5. $\beta(\mathrm{AD})=\beta(\mathrm{BC})+\theta 6=\left(40^{\circ} 00^{\prime} 32.78^{\prime \prime}+29^{\circ} 58^{\prime} 17.11^{\prime \prime}\right)=$ $=69^{\circ} 58^{\prime} 49.89^{\prime \prime}$.

\section{Coordinates of points}

Distance between two points (A and B) equals to 


$$
=\sqrt{\left(X_{2}-X_{1}\right)^{2}+\left(Y_{2}-Y_{1}\right)^{2}}
$$

$=\sqrt{(507,450.410-507,327.641)^{2}+(92,079.322-91,756.439)^{2}}$ $A B=345.4354 m$

$$
\frac{A B}{\operatorname{Sin}\left(\theta_{3}\right)}=\frac{B C}{\operatorname{Sin}\left(\theta_{1}\right)}
$$

$B C=\left(345.435 X \operatorname{Sin} 19^{\circ} 11^{\prime} 27.2^{\prime \prime}\right) / \operatorname{Sin} 43^{\circ} 11^{\prime} 26.59^{\prime \prime}=165.905 m$

$$
\frac{B C}{\operatorname{Sin}\left(\theta_{3}\right)}=\frac{A C}{\operatorname{Sin}\left(\theta_{2}\right)}
$$

$A C=\left(345.435 X \operatorname{Sin} 117^{\circ} 37^{\prime} 6.2^{\prime \prime}\right) / \operatorname{Sin} 43^{\circ} 11^{\prime} 26.59^{\prime \prime}=447.197 m$

$$
\frac{A D}{\operatorname{Sin}\left(\theta_{4}\right)}=\frac{A C}{\operatorname{Sin}\left(\theta_{5}\right)}
$$

$A D=\left(447.197 X \operatorname{Sin} 48^{\circ} 21^{\prime} 34.2^{\prime \prime} 10 / \operatorname{Sin} 101^{\circ} 40^{\prime} 8.80^{\prime \prime}=341.256 m\right.$

$$
\frac{C D}{\operatorname{Sin}\left(\theta_{6}\right)}=\frac{A D}{\operatorname{Sin}\left(\theta_{4}\right)}
$$

$C D=\left(341.256 X \operatorname{Sin} 29^{\circ} 58^{\prime} 17.11^{\prime \prime}\right) / \operatorname{Sin} 48^{\circ} 21^{\prime} 34.1^{\prime \prime}=228.120 m$

Easting of $\mathrm{C}=($ Easting of $\mathrm{A})+(\mathrm{AC} \times \operatorname{Sin} \beta(\mathrm{AC}))=$ $507,615.148 \mathrm{mE}$.

Northing of $\mathrm{C}=($ Northing of $\mathrm{A})+(\mathrm{AC} \times \mathrm{Cos} \beta(\mathrm{AC}))=$ $92,098.966 \mathrm{mN}$.

Easting of $\mathrm{C}=($ Easting of $\mathrm{B})+(\mathrm{BC} \times \operatorname{Sin} \beta(\mathrm{BC}))=$ $507,615.147 \mathrm{mE}$.

Northing of $\mathrm{C}=($ Northing of $\mathrm{B})+(\mathrm{BC} \times \operatorname{Cos} \beta(\mathrm{BC}))=$ $92,098.966 \mathrm{mN}$.

Easting of $\mathrm{D}=($ Easting of $\mathrm{A})+(\mathrm{AD} \times \operatorname{Sin} \beta(\mathrm{AD}))=$ $507,648.277 \mathrm{mE}$.

Northing of $\mathrm{D}=($ Northing of $\mathrm{A})+(\mathrm{AD} \times \operatorname{Cos} \beta(\mathrm{AD}))=$ $91,873.264 \mathrm{mN}$.

Easting of $\mathrm{D}=($ Easting of $\mathrm{C})+(\mathrm{CD} \times \operatorname{Sin} \beta(\mathrm{CD}))=$ $507,648.260 \mathrm{mE}$.

Northing of $\mathrm{D}=($ Northing of $\mathrm{C})+(\mathrm{CD} \times \operatorname{Cos} \beta(\mathrm{CD}))=$ $91,873.270 \mathrm{mN}$.

B. Adjustment of a double braced quadrilateral using least square condition equation

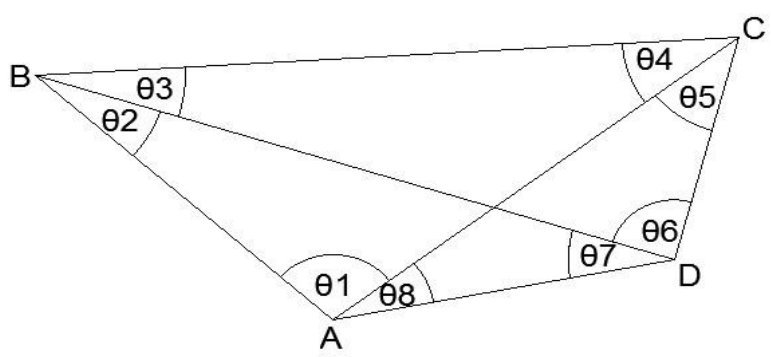

Fig. 2: Double Braced Quadrilateral

\begin{tabular}{|c|c|c|c|c|c|c|}
\hline $\begin{array}{c}\text { ANGLE } \\
(\theta)\end{array}$ & $\begin{array}{c}\text { STN } \\
\text { FROM }\end{array}$ & FACE & Degree & $\operatorname{mins}$ & $\mathrm{sec}$ & $\begin{array}{c}\text { STN } \\
\text { TO }\end{array}$ \\
\hline \multirow{4}{*}{8} & \multirow{4}{*}{ A } & $\mathrm{L}$ & 0 & 00 & 00 & $\mathrm{D}$ \\
\hline & & $\mathrm{L}$ & 29 & 58 & 15 & $\mathrm{C}$ \\
\hline & & $\mathrm{R}$ & 209 & 58 & 16 & $\mathrm{C}$ \\
\hline & & $\mathrm{R}$ & 180 & 00 & 05 & D \\
\hline \multirow{4}{*}{1} & \multirow{4}{*}{ A } & $\mathrm{L}$ & 10 & 00 & 00 & $\mathrm{C}$ \\
\hline & & $\mathrm{L}$ & 29 & 11 & 26 & B \\
\hline & & $\mathrm{R}$ & 209 & 11 & 25 & B \\
\hline & & $\mathrm{R}$ & 190 & 0 & 05 & $\mathrm{C}$ \\
\hline \multirow{4}{*}{2} & \multirow{4}{*}{ B } & $\mathrm{L}$ & 20 & 00 & 00 & $\mathrm{D}$ \\
\hline & & $\mathrm{L}$ & 84 & 39 & 23 & A \\
\hline & & $\mathrm{R}$ & 264 & 39 & 24 & $\mathrm{~A}$ \\
\hline & & $\mathrm{R}$ & 200 & 00 & 05 & D \\
\hline \multirow{4}{*}{3} & \multirow{4}{*}{ B } & $\mathrm{L}$ & 0 & 00 & 00 & D \\
\hline & & $\mathrm{L}$ & 52 & 57 & 42 & $\mathrm{C}$ \\
\hline & & $\mathrm{R}$ & 232 & 57 & 43 & $\mathrm{C}$ \\
\hline & & $\mathrm{R}$ & 180 & 00 & 05 & D \\
\hline \multirow{4}{*}{5} & \multirow{4}{*}{$\mathrm{C}$} & $\mathrm{L}$ & 0 & 00 & 00 & A \\
\hline & & $\mathrm{L}$ & 48 & 21 & 33 & B \\
\hline & & $\mathrm{R}$ & 228 & 21 & 34 & $\mathrm{~B}$ \\
\hline & & $\mathrm{R}$ & 180 & 00 & 05 & A \\
\hline \multirow{4}{*}{4} & \multirow{4}{*}{$\mathrm{C}$} & $\mathrm{L}$ & 10 & 00 & 00 & A \\
\hline & & $\mathrm{L}$ & 53 & 11 & 31 & $\mathrm{D}$ \\
\hline & & $\mathrm{R}$ & 233 & 11 & 32 & D \\
\hline & & $\mathrm{R}$ & 190 & 00 & 10 & A \\
\hline \multirow{4}{*}{7} & \multirow{4}{*}{$\mathrm{D}$} & $\mathrm{L}$ & 0 & 00 & 00 & B \\
\hline & & $\mathrm{L}$ & 66 & 10 & 57 & A \\
\hline & & $\mathrm{R}$ & 246 & 10 & 58 & A \\
\hline & & $\mathrm{R}$ & 180 & 00 & 04 & B \\
\hline
\end{tabular}

The measurements for a double braced quadrilateral $\mathrm{ABCD}$ are given below. Using this data, calculate the coordinates of station $\mathrm{C}$ and $\mathrm{D}$.

TABLE 5: FIELD OBSERVATION DATA 


\section{Angular Reduction AND CALCUlation OF StANDARd ERror $(\sigma)$}

TABLE 6: ANGULAR REDUCTION AND CALCULATION OF STANDARD ERRor $(\Sigma)$

\begin{tabular}{|c|c|c|c|c|c|c|c|}
\hline \multirow{2}{*}{$\begin{array}{c}\text { ANGLE } \\
(\theta)\end{array}$} & \multicolumn{2}{|c|}{$\theta$} & \multirow[b]{2}{*}{$\theta_{m}$} & \multicolumn{3}{|c|}{$\theta_{m}-\theta_{i}$} & \multirow{2}{*}{$\begin{array}{l}\text { S. E. } \\
(\sigma)\end{array}$} \\
\hline & $\mathrm{L}$ & $\mathrm{R}$ & & $\mathrm{V}=\theta_{m}-\theta_{l}$ & $\mathrm{~V}=\theta_{m}-\theta_{r}$ & $\left(\frac{\theta_{m}-\theta_{r}}{\theta_{m}-\theta_{l}}\right) / 2$ & \\
\hline 8 & $29^{\circ} 58^{\prime} 15^{\prime \prime}$ & $29^{\circ} 58^{\prime} 11^{\prime \prime}$ & $29^{\circ} 58^{\prime} 13.0^{\prime \prime}$ & 2.00 & 2.00 & $2.0^{\prime \prime}$ & 2.8 \\
\hline 1 & $19^{\circ} 11^{\prime} 26^{\prime \prime}$ & $19^{\circ} 11^{\prime} 20^{\prime \prime}$ & $19^{\circ} 11^{\prime} 23.0^{\prime \prime}$ & 3.00 & 3.00 & $3.00 \%$ & 4.2 \\
\hline 2 & $64^{\circ} 39^{\prime} 23^{\prime \prime}$ & $64^{\circ} 39^{\prime} 19^{\prime \prime}$ & $64^{\circ} 39^{\prime} 21.0^{\prime \prime}$ & 2.00 & 2.00 & $2.00^{\circ}$, & 2.8 \\
\hline 3 & $52^{\circ} 57^{\prime} 42^{\prime \prime}$ & $52^{\circ} 57^{\prime} 38^{\prime \prime}$ & $52^{\circ} 57^{\prime} 40.0^{\prime \prime}$ & 2.00 & 2.00 & $2.00^{\circ}$, & 2.8 \\
\hline 5 & $48^{\circ} 21^{\prime} 33^{\prime \prime}$ & $48^{\circ} 21^{\prime} 29^{\prime \prime}$ & $48^{\circ} 21^{\prime} 31.0^{\prime \prime}$ & 2.00 & 2.00 & $2.00^{\prime}$ & 2.8 \\
\hline 4 & $43^{\circ} 11^{\prime} 31^{\prime \prime}$ & $43^{\circ} 11^{\prime} 22^{\prime \prime}$ & $43^{\circ} 11^{\prime} 26.5^{\prime \prime}$ & 4.50 & 4.50 & $4.50^{\prime \prime}$ & 6.4 \\
\hline 7 & $66^{\circ} 10^{\prime} 57^{\prime \prime}$ & $66^{\circ} 10^{\prime} 54^{\prime \prime}$ & $66^{\circ} 10^{\prime} 55.5^{\prime \prime}$ & 1.50 & 1.50 & $1.50^{\prime \prime}$ & 2.1 \\
\hline 6 & $35^{\circ} 29^{\prime} 15^{\prime \prime}$ & $35^{\circ} 29^{\prime} 10^{\prime \prime}$ & $35^{\circ} 29^{\prime} 12.5^{\prime \prime}$ & 2.50 & 2.50 & $2.50^{\circ}$ & 3.5 \\
\hline
\end{tabular}

TABLE 7: OBSERVED ANGLES AND STANDARD ERRORS

\begin{tabular}{|c|c|c|c|c|c|}
\hline $\begin{array}{c}\text { ANGLE } \\
(\theta)\end{array}$ & $\begin{array}{c}\text { OBS. VALUE } \\
(\theta)\end{array}$ & $\begin{array}{c}\text { STD } \\
\text { ERROR } \\
\left(\sigma^{2}\right)\end{array}$ & STNn & $\mathrm{E}$ & $\mathrm{N}$ \\
\hline 1 & $19^{\circ} 11^{\prime} 23.0^{\prime \prime}$ & 4.2 & A & 507327.643 & 91756.449 \\
\hline 2 & $64^{\circ} 39^{\prime} 21.0^{\prime \prime}$ & 2.8 & B & 507450.402 & 92079.315 \\
\hline 3 & $52^{\circ} 57^{\prime} 40.0^{\prime \prime}$ & 2.8 & & & \\
\hline 4 & $43^{\circ} 11^{\prime} 26.5^{\prime \prime}$ & 6.4 & & & \\
\hline 5 & $48^{\circ} 21^{\prime} 31.0$ & 2.8 & & & \\
\hline 6 & $35^{\circ} 29^{\prime} 12.5^{\prime \prime}$ & 3.5 & & & \\
\hline 7 & $66^{\circ} 10^{\prime} 55.5^{\prime \prime}$ & 2.1 & & & \\
\hline 8 & $35^{\circ} 29^{\prime} 12.5^{\prime \prime}$ & 2.8 & & & \\
\hline
\end{tabular}

The number of total observations $(n)=8$ Angles.

The number of necessary observations $($ no) $=2$ (No of new points $)=2(2)=4$.

Hence, the number of conditions $(r)=n-n o=4$.

1. Condition equations

There are four condition equation:

1) Triangular condition equations are:

Fig. 2: ABCD

* Opposites angles $(\theta 7+\alpha 8)=(\theta 3+\theta 4)$

Also $(\theta 7+\mathrm{v} 7+\theta 8+\mathrm{v} 8)=(\theta 3+\mathrm{v} 3+\theta 4+\mathrm{v} 4)$

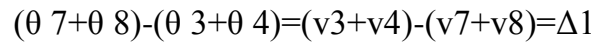

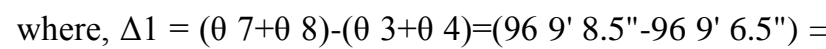
$=2.0^{\prime \prime}$

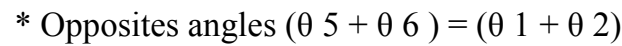

Also $(\theta 5+\mathrm{v} 5+\theta 6+\mathrm{v} 6)=(\theta 1+\mathrm{v} 1+\theta 2+\mathrm{v} 2)$

$(\theta 5+\theta 6)-(\theta 1+\theta 2)=(\mathrm{v} 1+\mathrm{v} 2)-(\mathrm{v} 5+\mathrm{v} 6)=\Delta 2$

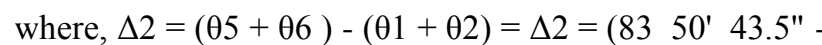
$\left.8350^{\prime} 44.0^{\prime \prime}\right)=-0.5^{\prime \prime}$.

Sum of angles in Fig. 2 ABCD: $v 1+v 2+v 3+v 4+v 5+v 6+v 7+v 8=\Delta 3$

where $\Delta 3=360^{\circ}-(\theta 1+\theta 2+\theta 3+\theta 4+\theta 5+\theta 6+\theta 7+\theta 8) \Delta 3=17.5^{\prime \prime}$

2) Side condition equations are:

Taking intersection of two diagonals as a pole for the figure $\mathrm{ABCD}$ :

$\delta 1 \mathrm{v} 1-\delta 2 \mathrm{v} 2+\delta 3 \mathrm{v} 3-\delta 4 \mathrm{v} 4+\delta 5 \mathrm{v} 5-\delta 6 \mathrm{v} 6+\delta 7 \mathrm{v} 7-\delta 8 \mathrm{v} 8=\Delta 4$ where:

$\Delta 4=$ Log $\sin (\theta 1)+$ Log $\sin (\theta 3)+\log \sin (\theta 5)+\log \sin (\theta 7)-\log$ $\sin (\theta 2)-\log \sin (\theta 4)-\log \sin (\theta 6)-\log \sin (\theta 8)=-1.213 \mathrm{E}-6$ $\delta \mathrm{i}$ is the difference in $\log \sin (\theta \mathrm{i})$ for a one second arc,

$$
\delta i=[\log \operatorname{Sin}(\theta+1 ")-\log \operatorname{Sin}(\theta)] \times 10-6
$$

TABLE 8: LOG SIN OF OBSERVATIONS

\begin{tabular}{|c|c|c|c|c|}
\hline $\begin{array}{c}\text { ANGLE } \\
(\theta)\end{array}$ & $\begin{array}{c}\text { OBS. } \\
\text { VALUE }(\theta)\end{array}$ & $\log \operatorname{Sin}(\theta+1)$ & $\log \operatorname{Sin}(\theta)$ & $\begin{array}{c}\delta \mathrm{i}\left(\mathrm{x} 10^{-}\right. \\
6\end{array}$ \\
\hline 1 & $\begin{array}{c}19^{\circ} 11^{\prime} \\
23.0^{\prime \prime}\end{array}$ & -0.483197895 & -0.483203945 & 6.050 \\
\hline 2 & $\begin{array}{c}64^{\circ} 39^{\prime} \\
21.0^{\prime \prime}\end{array}$ & -0.043949323 & -0.04395032 & 0.997 \\
\hline 3 & $\begin{array}{c}52^{\circ} 57^{\prime} \\
40.0^{\prime \prime}\end{array}$ & -0.097872079 & -0.097873668 & 1.589 \\
\hline 4 & $\begin{array}{c}43^{\circ} 11^{\prime} \\
26.5^{\prime \prime}\end{array}$ & -0.164669543 & -0.164671786 & 2.243 \\
\hline 5 & $\begin{array}{c}48^{\circ} 21^{\prime} \\
31.0^{\prime \prime}\end{array}$ & -0.126492515 & -0.126494387 & 1.872 \\
\hline 6 & $\begin{array}{c}35^{\circ} 29^{\prime} \\
12.5^{\prime \prime}\end{array}$ & -0.236183256 & -0.236186209 & 2.953 \\
\hline 7 & $\begin{array}{c}66^{\circ} 10^{\prime} \\
55.5^{\prime \prime}\end{array}$ & -0.038657043 & -0.038657972 & 0.929 \\
\hline \multirow{2}{*}{$\begin{array}{c}29^{\circ} 58^{\prime} \\
13.0^{\prime \prime}\end{array}$} & -0.301416793 & -0.301420444 & 3.651 \\
\hline
\end{tabular}

$\left[\begin{array}{cccccccc}1 & 0 & 0 & -1 & -1 & 0 & 0 & 1 \\ 0 & -1 & -1 & 0 & 0 & 1 & 1 & 0 \\ 1 & 1 & 1 & 1 & 1 & 1 & 1 & 1 \\ 6.05 \times 10^{-6} & 0.997 \times 10^{-6} & 1.589 \times 10^{-6} & 2.243 .05 \times 10^{-6} & 1.872 \times 10^{-6} & 2.953 \times 10^{-6} & 0.929 \times 10^{-6} & 3.651 \times 10^{-6}\end{array}\right]$




$$
\Delta=\left[\begin{array}{c}
2 \\
-0.5 \\
17.5 \\
-1.213 \times 10^{-6}
\end{array}\right]
$$

which is in the general form $\mathrm{Bv}=\Delta$ and the weight matrix is:

$\mathrm{W}=\operatorname{diag} .\left[1 / \sigma^{2}{ }_{1} 1 / \sigma^{2}{ }_{2} 1 / \sigma^{2}{ }_{3} 1 / \sigma^{2}{ }_{4} 1 / \sigma^{2}{ }_{5} 1 / \sigma^{2}{ }_{6} 1 / \sigma^{2}{ }_{7} 1 / \sigma^{2}{ }_{8}\right]$

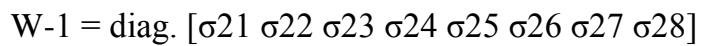

$$
W^{-1}=\left[\begin{array}{cccccccc}
17.64 & 0 & 0 & 0 & 0 & 0 & 0 & 0 \\
0 & 7.84 & 0 & 0 & 0 & 0 & 0 & 0 \\
0 & 0 & 7.84 & 0 & 0 & 0 & 0 & 0 \\
0 & 0 & 0 & 40.96 & 0 & 0 & 0 & 0 \\
0 & 0 & 0 & 0 & 7.84 & 0 & 0 & 0 \\
0 & 0 & 0 & 0 & 0 & 12.25 & 0 & 0 \\
0 & 0 & 0 & 0 & 0 & 0 & 4.41 & 0 \\
0 & 0 & 0 & 0 & 0 & 0 & 0 & 7.84
\end{array}\right]
$$

2. The normal equations is given as:

$$
\begin{gathered}
M=B W^{-1} B^{T} \\
M=\left[\begin{array}{llll}
74.2800 & 0 & -23.3200 & 0.0000 \\
0 & 32.3400 & 0.9800 & 0.0000 \\
-23.3200 & 0.9800 & 106.6200 & 0.0003 \\
0.0000 & 0.0000 & 0.0003 & 0.0000
\end{array}\right]
\end{gathered}
$$

3. The Lagrange multipliers are:

$$
K=\left[\begin{array}{llll}
74.2800 & 0 & -23.3200 & 0.0000 \\
0 & 32.3400 & 0.9800 & 0.0000 \\
-23.3200 & 0.9800 & 106.6200 & 0.0003 \\
0.0000 & 0.0000 & 0.0003 & 0.0000
\end{array}\right]^{-1}\left[\begin{array}{c}
2 \\
-0.5 \\
17.5 \\
-1.213 \times 10^{-6}
\end{array}\right]
$$$$
K=\left[\begin{array}{l}
0.0000 \\
0.0000 \\
0.0000 \\
-4.6851
\end{array}\right] \times 10^{5}
$$$$
V=W^{-1} B^{T} K
$$$$
V=\left[\begin{array}{c}
-8.0783^{\prime \prime} \\
7.5157^{\prime \prime} \\
5.3412^{\prime \prime} \\
-5.2204^{\prime \prime} \\
0.3635^{\prime \prime} \\
6.0111^{\prime \prime} \\
6.3458^{\prime \prime} \\
5.2214^{\prime \prime}
\end{array}\right]
$$

\begin{tabular}{|c|c|c|c|}
\hline $\begin{array}{c}\text { ANGLE } \\
(\theta)\end{array}$ & $\begin{array}{c}\text { OBSERVED } \\
\text { ANGLE } \\
(\theta)\end{array}$ & $\begin{array}{c}\text { Residuals } \\
\text { (v) }\end{array}$ & $\begin{array}{c}\text { CORRECTED } \\
\text { ANGLE } \\
(\theta)\end{array}$ \\
\hline 1 & $19^{\circ} 11^{\prime} 23.0^{\prime \prime}$ & $-8.0783 "$ & $19^{\circ} 11^{\prime} 14.9217^{\prime \prime}$ \\
\hline 2 & $64^{\circ} 39^{\prime} 21.0^{\prime \prime}$ & $7.5157 "$ & $64^{\circ} 39^{\prime} 28.5157^{\prime \prime}$ \\
\hline 3 & $52^{\circ} 57^{\prime} 40.0^{\prime \prime}$ & $5.3412^{\prime \prime}$ & $52^{\circ} 57^{\prime} 45.3412^{\prime \prime}$ \\
\hline 4 & $43^{\circ} 11^{\prime} 26.5^{\prime \prime}$ & $-5.2204 "$ & $43^{\circ} 11^{\prime} 21.276^{\prime \prime}$ \\
\hline 5 & $48^{\circ} 21^{\prime} 31.0^{\prime \prime}$ & $0.3635^{\prime \prime}$ & $48^{\circ} 21^{\prime} 31.3635^{\prime \prime}$ \\
\hline 6 & $35^{\circ} 29^{\prime} 12.5^{\prime \prime}$ & $6.0111 "$ & $35^{\circ} 29^{\prime} 18.5111^{\prime \prime}$ \\
\hline 7 & $66^{\circ} 10^{\prime} 55.5^{\prime \prime}$ & $6.3458 "$ & $66^{\circ} 11^{\prime} 01.8458^{\prime \prime}$ \\
\hline 8 & $29^{\circ} 58^{\prime} 13.0^{\prime \prime}$ & $5.2214 "$ & $29^{\circ} 58^{\prime} 18.2214^{\prime \prime}$ \\
\hline$\Sigma$ & $35959^{\prime} 42.5^{\prime \prime}$ & $17.5^{\prime \prime}$ & $360^{\circ} 00^{\prime} 0.00^{\prime \prime}$ \\
\hline
\end{tabular}

\section{The adjusted observations}

Finally, adding the residuals to the given observations, we obtain the adjusted observations: $\mathrm{L}=\mathrm{L}+\mathrm{v}$.

TABLE 9: FINAL ADJUSTED OBSERVATIONS

5. Azimuth of lines:

Bearing of line, $\beta=\tan -1(\mathrm{X} 2-\mathrm{X} 1) /(\mathrm{Y} 2-\mathrm{Y} 1)$

$\beta(A B)=\tan ^{-1} \quad(507,450.402-507,327.643) /(92,079.315$ $91,756.443)=20^{\circ} 49^{\prime} 5.58^{\prime \prime}$

$\operatorname{ANGLE}(\theta 2+\theta 3)=64^{\circ} 39^{\prime} 28.52^{\prime \prime}+52^{\circ} 57^{\prime} 45.34^{\prime \prime}=117^{\circ} 37^{\prime}$ $13.84 "$

$\beta(B C)=20^{\circ} 49^{\prime} 5.58^{\prime \prime}+180-117^{\circ} 37^{\prime} 13.84^{\prime \prime}=83^{\circ} 11^{\prime}$ $51.74 "$

ANGLE $(\theta 4+\theta 5)=43^{\circ} 11^{\prime} 21.28^{\prime \prime}+48^{\circ} 21^{\prime} 31.36^{\prime \prime}=91^{\circ} 32^{\prime}$ $52.64 " \beta(C D)=83^{\circ} 11^{\prime} 51.74^{\prime \prime}+180-91^{\circ} 32^{\prime} 52.64^{\prime \prime}=171^{\circ}$ $38^{\prime} 59.10^{\prime \prime}$

$\operatorname{ANGLE}(\theta 6+\theta 7)=35^{\circ} 29^{\prime} 18.51^{\prime \prime}+66^{\circ} 11^{\prime} 01.85^{\prime \prime}=101^{\circ}$ $40^{\prime} 20.36^{\prime \prime} \beta(\mathrm{DA})=171^{\circ} 38^{\prime} 59.10^{\prime \prime}+180-101^{\circ} 40^{\prime} 20.36^{\prime \prime}$ $=249^{\circ} 58^{\prime} 38.74^{\prime \prime}$

$\operatorname{ANGLE}(\theta 8+\theta 1)=29^{\circ} 58^{\prime} 18.22^{\prime \prime}+19^{\circ} 11^{\prime} 14.92^{\prime \prime}=49^{\circ}$ 9' 33.14" $\beta(\mathrm{AD})=20^{\circ} 49^{\prime} 5.58+49^{\circ} \quad 9^{\prime} \quad 33.14^{\prime \prime}=69^{\circ} 58^{\prime}$ $38.72 "$

$\operatorname{ANGLE}(\theta 1)=19^{\circ} 11^{\prime} 14.92^{\prime \prime}$

$\beta(A C)=20^{\circ} 49^{\prime} 5.58^{\prime \prime}+19^{\circ} 11^{\prime} 14.92^{\prime \prime}=40^{\circ} 00^{\prime} 20.50^{\prime \prime}$

$\beta(\mathrm{BD})=20^{\circ} 49^{\prime} 5.58^{\prime \prime}+180-64^{\circ} 39^{\prime} 28.52^{\prime \prime}=136^{\circ} 09^{\prime} 37.06^{\prime \prime}$

6. Coordinates of points

$$
\begin{gathered}
A B=\sqrt{\left(X_{2}-X_{1}\right)^{2}+\left(Y_{2}-Y_{1}\right)^{2}} \\
A B=\sqrt{(507,450.410-507,327.641)^{2}+(92,079.322-91,756.439)^{2}} \\
A B=345.4354 m \\
\frac{B C}{\operatorname{Sin}\left(\theta_{1}\right)}=\frac{A B}{\operatorname{Sin}\left(\theta_{4}\right)} \quad A C=A B \operatorname{Sin}\left(\theta_{1}\right) / \operatorname{Sin}\left(\theta_{4}\right) \\
B C=\left(345.435 X \operatorname{Sin} 19^{\circ} 11^{\prime} 14.92\right) / \operatorname{Sin} 43^{\circ} 11^{\prime} 21.28^{\prime \prime} \\
B C=165.881 m
\end{gathered}
$$




$$
\begin{gathered}
\frac{A C}{\operatorname{Sin}\left(\theta_{2}+\theta_{3}\right)}=\frac{A B}{\operatorname{Sin}\left(\theta_{4}\right)} \\
A C=A B \operatorname{Sin}\left(\theta_{2}+\theta_{3}\right) / \operatorname{Sin}\left(\theta_{4}\right)
\end{gathered}
$$

$A C=\left(345.435 X \operatorname{Sin} 117^{\circ} 37^{\prime} 13.86^{\prime \prime}\right) / \operatorname{Sin} 43^{\circ} 11^{\prime} 21.28^{\prime \prime}$

$$
A C=447.200 m
$$

$$
\frac{B D}{\operatorname{Sin}\left(\theta_{1}+\theta_{8}\right)}=\frac{A B}{\operatorname{Sin}\left(\theta_{7}\right)}
$$

$B D=A B \operatorname{Sin}\left(\theta_{1}+\theta_{8}\right) / \operatorname{Sin}\left(\theta_{7}\right)$

$B D=\left(345.435 X \operatorname{Sin} 49^{\circ} 09^{\prime} 33.14^{\prime \prime}\right) / \operatorname{Sin} 66^{\circ} 11^{\prime} 1.85^{\prime \prime}$

$$
B D=285.657 m
$$

$$
\frac{A C}{\operatorname{Sin}\left(\theta_{7}+\theta_{6}\right)}=\frac{A D}{\operatorname{Sin}\left(\theta_{5}\right)}
$$

$$
\mathrm{A} D=A C \operatorname{Sin}\left(\theta_{5}\right) / \operatorname{Sin}\left(\theta_{7}+\theta_{6}\right)
$$

$A D=\left(447.20 X \operatorname{Sin} 48^{\circ} 21^{\prime} 31.36^{\prime \prime}\right) / \operatorname{Sin} 101^{\circ} 40^{\prime} 20.37^{\prime \prime}$

$$
A D=341.258 m
$$

Easting of $C=($ Easting of $\mathrm{A})+(A C x \operatorname{Sin} \beta(A C))=507,615.123 m E$

Northing of $C=($ Easting of $\mathrm{A})+(A C \times \operatorname{Cos} \beta(A C))=92,098.985 \mathrm{mN}$

Easting of $D=($ Easting of $\mathrm{A})+(\mathrm{AD} x \operatorname{Sin} \beta(A D))=507,648.273 m E$

Northing of $D=($ Northing of $\mathrm{A})+(C D \times \operatorname{Cos} \beta(C D))=91,873.282 \mathrm{mN}$

Easting of $C=($ Easting of $B)+(B C x \operatorname{Sin} \beta(B C))=507,615.123 m E$

Northing of $C=($ Northing of $B)+(B C x \operatorname{Cos} \beta(B C))=92,098.970 \mathrm{mN}$

Easting of $D=($ Easting of $\mathrm{B})+(\mathrm{AD} x \operatorname{Sin} \beta(A D))=507,648.268 m E$

Northing of $D=($ Northing of $\mathrm{B})+(C D \times \operatorname{Cos} \beta(C D))=91,873.283 \mathrm{mN}$

\section{CONCLUSION}

Comparisons of the solutions derived from Observation 1 and Observation 2 showed a high degree of agreement. Therefore, since the solutions from the adjustment of the single and double brace quadrilateral are the same we conclude that the adjustment is a reliable. In summary, some of the primary conditions for LS adjustment among others are that:

(i) the number of field observations must exceed the number of parameters to be determined;

(ii) the number of observation equations formed must be equal to the number of field observations;

(iii) the number of condition equations formed must equal the difference between the number of observations and the number of unknown parameters to be determined.

\section{REFERENCES}

[1] Ayeni, O. O., (2001). Statistical adjustment and analysis of data. A Manual, in the Department of Surveying \& Geoinformatics, Faculty of Engineering, University of Lagos, Nigeria, 2001

[2] Oladosu S. O. Okonofua S. E. and Ehigiator-Irughe R.3. (2019) Implementation of Condition Equation Model in Geodetic Observation: A Case Study of Circular Reservoir Structure. Nigerian Journal of Environmental Sciences and Technology (NIJEST. Vol 3 No. 2 October 2019, pp $233-244$.

[3] Ehigiator - Irughe, R. and Ehigiator M. O., (2010). Estimation of the centre coordinates and radius of Forcados Oil Tank from Total Station data using least square Analysis" International Journal of pure and applied sciences. A pan - African Journal Series.

[4] Ehigiator - Irughe, R. Ashraf A. A. Beshr, J. O. Ehiorobo and O.M Ehigiator (2011). Modification of Geodetic Methods for Determining the Monitoring Station Coordinates on the Surface of Cylindrical Oil Storage Tank. Research Journal of Engineering and Applied Sciences (RJEAS) 1 (1) pp. 58 - 63. A United State Academy publications USA

[5] Ehigiator - Irughe, R. Ashraf A. A. Beshr, J. O. Ehiorobo and O.M. Ehigiator (2011). Modification of Geodetic Methods for Determining the Monitoring Station Coordinates on the Surface of Cylindrical Oil Storage Tank. Research Journal of Engineering and Applied Sciences (RJEAS) 1 (1) pp. 58 - 63. A United State Academy publications USA

[6] Ehigiator-Irughe R., Ehiorobo J. O., Ashraf A. B. and. Ehigiator M. O., (2012). Determination of the Ovality of Crude Oil Storage Tanks using Least Squares. Advanced Materials Research Vol. 367 (2012) pp 475 483C (2012) Trans Tech Publications, Switzerland doi:10.4028/www.scientific.net/AMR.367.475. 